Revista de Psicología de la PUCP. Vol. XIV. No 1. 1996

\title{
HACIA UN ANALISIS COGNITIVO DEL CAMBIO CONDUCTUAL: EL COMPORTAMIENTO SOCIAL PRO ACTIVO
}

Manolete S. Moscoso'

\begin{abstract}
Este artículo presenta un análisis del Comportamiento Social Pro Activo utilizando como modelo la teoría del aprendizaje social. Se revisa el concepto de autoeficacia, originalmente propuesto por Bandura, y se hace referencia a variables de tipo cognitivo tales como los sistemas de valores y creencias. Se examinan las distinciones entre el temor al fracaso, al éxito, al rechazo, y su relación con el proceso de autosabotaje. Asimismo se discute la importancia de la transformación del significado y sus beneficios en la extinción del temor. Se presenta un modelo conceprual del control de los estados emocionales y sus implicancias en el proceso de la acción. Finalmente, se describen las premisas fundamentales del Condicionamiento Neurofisiológico Asociativo como un programa terapéutico que facilita el manejo de los estados emocionales de la persona.

Palabras Claves: Comportamiento social pro activo, autoeficacia, cambio conductual, condicionamiento neurofisiológico asociativo.
\end{abstract}

Toward a cognitive analysis of behavioral change: the pro active social behavior

This article presents an analysis of the pro active social behavior construct based on the social learning theory. Self efficacy is briefly reviewed and discussed in the context of Bandura's conceptual work. Reference is made to cognitive variables such as values and beliefs, as fundamental principles in the development of the pro active social behavior. I examine the distinctions between fears of failure, success, and rejection as they relate to the process of self sabotage. Comments are made on the transformation of meaning and its benefits on fear extinction. Finally, I briefly describe the principles of the associative neurophysiological conditioning as a therapeutic program for the management of emotional states by the individual.

Keywords: Pro active social behaviour, behavioral change, neurophysiological associative conditioning.

1 Psicólogo graduado en la Universidad Nacional Mayor de San Marcos en Lima, Perú; realizó sus estudios doctorales en la Universidad de California, Los Angeles (UCLA) y la Universidad Sierra. Autor de varios artículos de investigación publicados en las áreas de Estrés, "Biofeedback", y Psicología de la Salud. Acrualmente, es director del Instituto Stress \& Cognition Research en Tampa, Florida, Profesor Visitante de la Universidad Intercontinental de la ciudad de México, Psicólogo del Centro de Investigación en Medicina Conductual y Psicología de la Salud de la Universidad del Sur de la Florida.

Dirección: 15826 Spring Crest Circle, Tampa, Florida, 33624 



\section{Introducción}

Un gran número de estudios de investigación en el campo de la psicoterapia, nos han mostrado que las personas logran obtener resultados favorables en cuanto a un cambio conductual con la ayuda de tratamientos profesionales (Lambert, Shapiro, y Bergin, 1986; Seligman, 1995); sin embargo, estos mismos estudios nos enseñan muy poco acerca de como se produce tal cambio.

La literatura también menciona estudios de investigación que reportan sobre personas con problemas de conducta, que logran cambiar su estilo de vida sin necesidad de recibir tratamiento psicológico (Marlett, Baer, Donovan, y Divlahan, 1988); pero estos estudios no indican cómo esos individuos son capaces de lograr resultados positivos por sus propios medios.

El propósito de los estudios de investigación en psicoterapia es demostrar que las personas pueden cambiar conductas o estilos de vida indeseables. Estos trabajos reportan que muchos individuos cambian y luego vuelven a recaer, o que el proceso de cambio en algunos toma un tiempo demasiado largo y que, por último, otros no logran cambiar en absoluto. Debido a estas inconsistencias sobre cambio conductual reportadas en la literatura psicológica, encuentro justificado el preguntarnos por las razones de estas diferencias individuales.

Mi profundo interés por el campo de la psicología de la salud y sus implicancias en el estilo de vida de una persona, me lleva a concentrar esfuerzos en tratar de entender esa estructura necesaria para el cambio conductual, y su mantenimiento a largo plazo. La pregunta fundamental puede ser presentada de la siguiente manera: ¿Cuáles son las condiciones necesarias para crear un cambio conductual en el individuo? 
El objetivo del presente trabajo es tratar de responder a esta pregunta, así como también ofrecer puntos de vista susceptibles de evaluación empírica. Responder a esta pregunta implica hacer un análisis de factores motivacionales, conscientes y fuera de la conciencia, así como de ciertos aspectos de interpretación cognitiva que limitan la utilización de recursos y habilidades personales por parte del individuo que conduzcan a lograr este cambio.

Con este propósito, voy a asumir las siguientes hipótesis de trabajo: (1) La conducta humana está dirigida en base a metas. (2) Los logros de un individuo no están en proporción a su potencial y a sus recursos.

La habilidad de llegar a ser consciente de esta diferencia en términos de expectativas de autoeficacia y expectativas de resultados y facilitar un sentido de congruencia, es decir, crear un estilo de vida en base al potencial real y recursos personales, es una de las premisas básicas en el desarrollo del Comportamiento Social Pro Activo al cual nos referiremos con más detalle en las páginas que siguen.

Figura 1: Representación de la diferencia entre expectativas de autoeficacia y expectativas de resultados

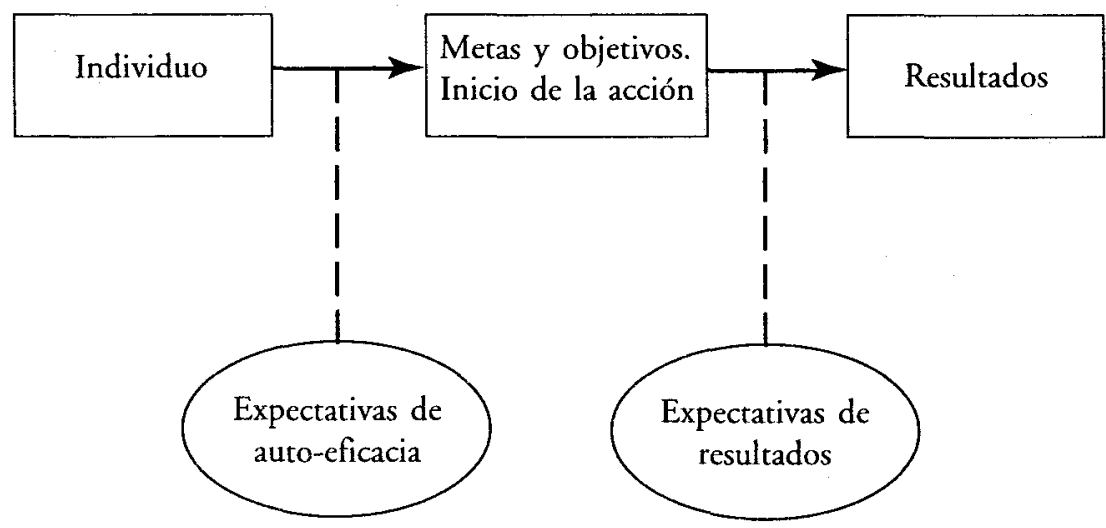




\section{El comportamiento social pro activo}

El Comportamiento Social Pro Activo (CSPA) es el conjunto de actitudes, hábitos y conductas que promueven y estimulan la salud de un individuo (Moscoso, 1994). Entiéndase éste como un patrón conductual, el cual está representado por un conjunto de valores y creencias adscritos a un estilo de vida que tiene como prioridad la salud y la búsqueda de un sentido de equilibrio mental y físico.

En el proceso de condicionamiento que conduce a la adquisición del CSPA, el individuo tiene que hacer uso de sus habilidades. En este sentido, el optimismo juega un rol básico. De acuerdo con un buen número de estudios de investigación en el campo del estrés, la persona optimista reporta buenos estados de salud en forma general (Scheier y Carver, 1992). Además, el optimismo puede ser considerado como un factor significativo para predecir una pronta recuperación de ciertas enfermedades (Sheier et. al., 1989).

Uno de los factores motivacionales más significativos para la adquisición del CSPA, es la creencia de que uno intenta y desea un mayor desarrollo personal. Esto, a su vez, implica ser una persona de acción, es decir, alguien que aspira a un estilo de vida óptimo y saludable; implica situarse en un estado mental de fe total y absoluta expectativa de que uno puede lograr todo lo que uno desea, ahora.

El CSPA considera al inicio de la acción como un elemento esencial para obtener resultados positivos y como una vía eficaz en la autoregulación de los estados emocionales y de la conducta. El CSPA es el resultado de actuar en forma consistente y objetiva a través de un plan previamente diseñado con el propósito de lograr un estilo de vida óptimo y saludable. La premisa fundamental del concepto de acción se basa en las interpretaciones que el individuo hace acerca de sus expectativas de autoeficacia, en función a sus intenciones, metas y planes. Este proceso implica tres fases:

\section{Conocer claramente el objetivo}

Cuanto más claro y específico sea este objetivo, su alcance será más probable. En este sentido, claridad y objetividad son fundamentales, de 
tal manera que uno llegue a saber cuáles son las fuerzas que motivan e impulsan su conducta. Para lograr este estado es importante preguntarse acerca de la dirección que uno busca, si realmente desea insistir.

Consideremos, por ejemplo, el caso de una persona que sufre de obesidad, a quien se le ha recomendado la reducción del consumo de grasas y calorías en su dieta diaria. Si esta persona conceptúa la reducción de grasas y calorías como objetivo, es muy posible que no logre una pérdida de peso o, si lo consigue, será temporal. Para que realmente se produzca un cambio, esta persona necesitará en parte modificar su estilo de vida que incluya, además, un programa de ejercicios aeróbicos en forma regular. Por lo tanto el objetivo principal en este caso es la búsqueda de equilibrio entre reducción en el consumo de calorías y grasas, así como un aumento de entrenamiento aeróbico.

La programación de metas y objetivos es vital en la adquisición del CSPA. Este primer concepto es vagamente entendido por adolescentes, quienes están más interesados en "vivir el presente". A su vez, es largamente ignorado por la gran mayoría de adultos, quienes están más preocupados por "los problemas de la vida diaria". Está basado en la suposición de que, obviamente, para obtener resultados positivos hay que tener un plan de acción. Sin embargo, olvidamos que este plan requiere de un proceso de organización detallado que involucre todos los pasos a seguir en forma específica.

Muchos planes no son culminados con éxito por la simple razón de que no se programaron los objetivos y tampoco se elaboró un minucioso plan de acción. Particularmente en nuestra cultura latinoamericana, este es un problema serio no solamente a nivel individual sino, también, a nivel empresarial, comunitario y de gobierno.

El programar metas y objetivos significa crear un futuro por adelantado. Cuando se planean metas, se desarrolla un plan de trabajo el cual permite adquirir una visión del futuro. Cuanto más detallados y mejor organizados son los planes, esta visión del futuro es más clara. Por otro lado, la diferencia entre "soñar despierto"y crear un futuro firme, está en la dosis de acción que se pone en el proceso de alcanzar los objetivos propuestos. 
La creación de metas, particularmente las de largo plazo, viene a ser la base del proceso de iniciar acción. En esencia, esta programación de metas necesita ser considerada como más importante que el propio resultado. Esta es la gran diferencia entre metas que estimulan y metas que no lo hacen, siendo esta última distinción un aspecto importante en el desarrollo del CSPA.

Desde el punto de vista de la teoría social cognitiva, el poder motivacional de fijar metas deriva no solamente de las metas mismas, sino también de los procesos de autoregulación. Cuando una persona está trabajando con la intención de alcanzar una meta específica, tiende a evaluar la relación de su propio rendimiento y sus logros (Bandura y Cervone, 1983). Este proceso de retroalimentación es la base de la motivación autodirigida, la cual incluye evaluaciones de la eficacia del propio rendimiento (Carver y Scheier, 1990).

El hecho de autoevaluarse en relación a su propia actuación y rendimiento es un fenómeno natural en la programación de metas. Cuando uno evalúa positivamente los logros parciales, esto sirve a la vez de reforzador para mayores logros hasta alcanzar la meta final. Cuando sucede lo opuesto, es decir una evaluación negativa, ésta no solamente pone en peligro el éxito de la meta final, sino que puede ocasionar estados emocionales negativos (Ahrens, 1987).

En el caso de algunos individuos, la insatisfacción en cuanto al logro de resultados puede servir de estímulo para un mayor esfuerzo (Bandura y Cervone, 1986). Considerar la satisfacción personal como contingente para un mayor esfuerzo y logros mayores puede ser un poderoso motivador para que una persona logre alcanzar las metas propuestas a largo plazo (Bandura, 1989).

Otro factor motivacional muy importante en el proceso de alcanzar metas es la percepción de autoeficacia (Bandura, 1986). La actuación y rendimiento estable hacia el logro de una meta, permite a la persona adquirir un sentimiento de mayor confianza, es decir, que la meta es alcanzable desde todo punto de vista. Por lo general, individuos con un fuerte sentido de autoeficacia planifican sus metas de una manera mucho más organizada y a un nivel más elaborado (Wood, Bandura, y Bailey, 1990). 
La planificación de metas y objetivos es un proceso constante, metódico y específico que requiere de revisión periódica en forma sistemática. Sin este primer paso, es muy difícil mantener un plan de acción realmente efectivo.

\section{Acción}

Una vez establecido el objetivo o meta, el paso siguiente es iniciar la acción y actuar en pro de la obtención de éste. Ciertamente el individuo tiene que tomar la decisión de actuar, y esto ocurre en base a la creencia de que determinado aspecto en la vida de una persona, institución, o comunidad requiere, en alguna medida de un mejoramiento. Este concepto es entendido como intencionalidad y deseo.

La intencionalidad en el inicio de la acción implica una diferenciación clara entre los medios y los fines. Considérese este concepto como otro factor fundamental para el éxito en la adquisición del CSPA, así como también para el éxito en cualquier forma de un proceso psicoterapéutico.

\section{Evaluación de resultados parciales}

Se refiere al proceso de evaluar los resultados parciales que una persona viene obteniendo como consecuencia de la planificación de objetivos, decisiones y acciones tomadas hasta ese momento. Esta tercera fase necesita ser realizada de manera constante, lo que permite juzgar en forma objetiva si las decisiones y acciones que se han tomado en consideración funcionan, y si producen resultados parciales positivos que permitan acercarse a las metas finales.

Dentro de este proceso, es vital el concepto de flexibilidad, es decir mantenerse dispuesto a aceptar nuevas posibilidades y cambios que sean necesarios para aproximarse a la meta deseada. Esta situación de cambiar constantemente los planes en la búsqueda del camino que lleve a alcanzar la meta final, implica una interacción constante con el temor al fracaso. En este sentido, el desarrollo del CSPA a través de la acción, permite la adquisición de recursos y habilidades que permiten vencer este temor $y$ familiarizarse con estratégias cognitivas que facilitan el cambio conductual, deseado. 
Dentro de este esquema de cambio conductual es necesario aceptar que el conocimiento no es suficiente. Unicamente con conocimiento no se producen resultados positivos; para que ello ocurra es necesario el inicio de la acción. Por ejemplo, el hecho de saber que el consumo de tabaco causa cáncer pulmonar no es razón suficiente para que muchas personas dejen de fumar. Como podemos observar, la diferencia no radica en saber qué hacer, sino más bien en simplemente hacer lo que ya sabemos. Esta forma de pensar típica del CSPA facilita el afrontamiento exitoso del temor al fracaso.

Figura 2: Mecanismos del cambio conductual

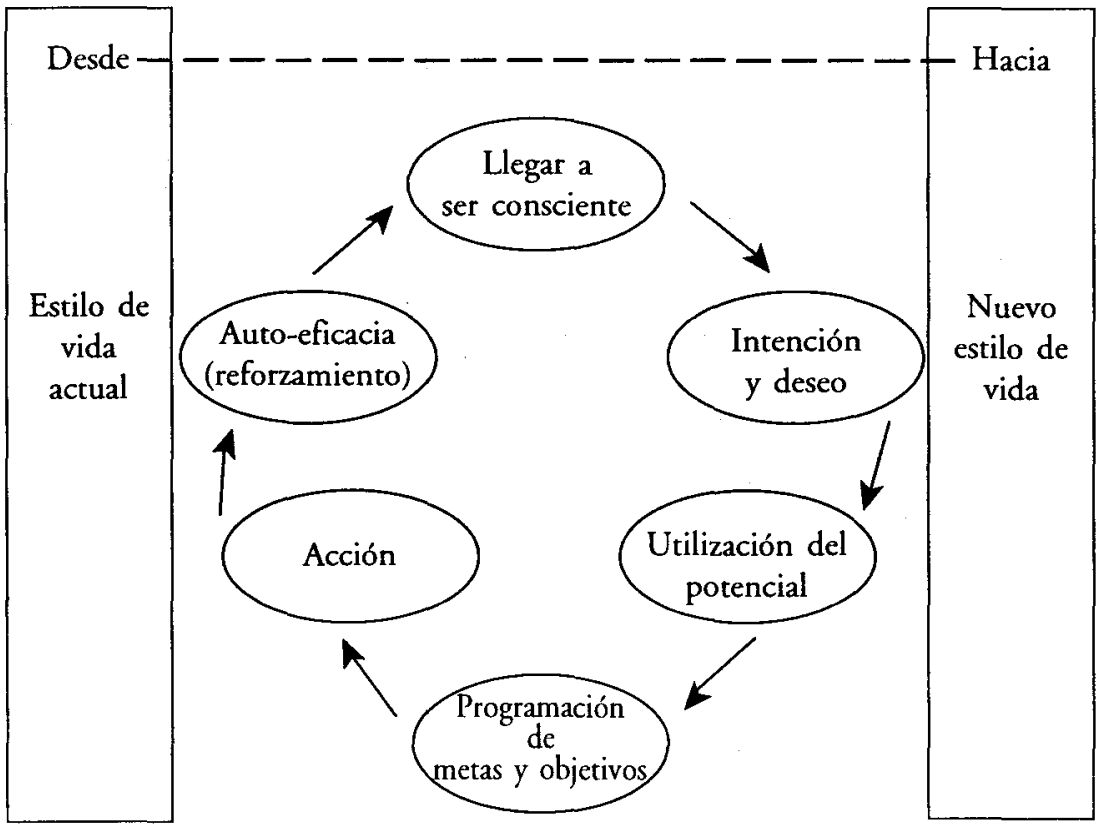

En su intento de explicar los procesos cognitivos mediadores del cambio conductual, la teoría social del aprendizaje, como marco teóricoconceptual del CSPA, también reconoce que la habilidad de un individuo de iniciar la acción viene a significar uno de los factores más poderosos en el proceso de cambio conductual. En este sentido, los procedimientos 
psicológicos con fines terapéuticos sirven como fuentes de refortalecimiento para las expectativas de autoeficacia de un individuo (Bandura, 1977a, Bandura, 1988b).

\section{El concepto de autoeficacia}

La literatura psicológica en el campo de la teoría social del aprendizaje, ha mostrado suficiente evidencia de que el cambio conductual depende, directamente, de métodos de empowerment o poder personal, en la medida que la persona dispone de un buen nivel de conocimientos, atributos, y creencias de autoeficacia que le permita ejercer control sobre sí mismo (Bandura, 1988a).

El concepto de autoeficacia está relacionado con la creencia del individuo en sus propios recursos y habilidades, los cuales le permiten movilizar un adecuado nivel de motivación para el inicio de un plan de acción destinado a ejercer un adecuado control sobre su conducta (Bandura y Adams, 1977). Asimismo, postula que las diferentes formas de influencia (acción, modelaje, persuación verbal, cambios psicofisiológicos), generan un cambio conductual a través del reforzamiento de las expectativas de autoeficacia (Bandura, Adams, y Beyer).

De acuerdo a esta formulación, la percepción de autoeficacia puede alterar y cambiar la conducta en diferentes formas, teniendo un efecto significativo en el desarrollo personal y estilo de vida (Bandura, 1989). En este sentido, es importante indicar que la autoeficacia debe de ser entendida como un factor vital y de mucha influencia en el desarrollo del CSPA, aunque no necesariamente el único de éste.

Las expectativas de autoeficacia, por parte de un individuo, refuerzan las posibilidades de actuar con el propósito de lograr una meta (Bandura, 1977a). Esta convicción estimula la creencia de que uno puede actuar con posibilidades de éxito y lograr los resultados deseados. Por ejemplo, en la medida en que una persona tenga la creencia de ser eficaz en eliminar su consumo de tabaco, ésta va a mostrarse interesada en iniciar un programa de tratamiento de esta naturaleza. La fuerza de convicción en su habilidad, va a servir de termómetro en cuanto a la posibilidad de iniciar tal acción. Cuanto más fuerte sea su percepción de autoeficacia, sus esfuerzos serán mayores. 
Sin lugar a dudas, este concepto es parte integral del CSPA. A la vez, es de gran utilidad para predecir el cambio conductual, particularmente dentro del contexto terapéutico. La autoeficacia no es un beneficio que se adquiere con la edad o color de la piel. Es un atributo que debe ser creado por el individuo; es un sentimiento de poder y creencia en la habilidad personal. El sentirse confiado y seguro en un determinado momento no es más que un estado mental creado por uno mismo. El reto es cómo mantener esta cualidad personal, de manera que tenga un efecto duradero y que favorezca la adquisición del CSPA con el propósito de gozar de un estilo de vida óptimo y saludable.

El CSPA es un patrón conductual que promueve la salud, por lo tanto es importante estimular el desarrollo de éste a nivel individual y comunitario, particularmente en culturas donde los conceptos de salud y conducta no están claramente entendidos o aceptados como el resultado de un conjunto específico de creencias y valores. En este sentido, considero que, a nivel de entrenamiento individual, las técnicas cognitivo-conductuales tienen la mejor opción de estimular su desarrollo.

Promover el CSPA a nivel comunitario es un reto de la salud pública, que requiere programas de cambio conductual en base a campañas dirigidas a modificar valores, actitudes y creencias de la comunidad en relación a la salud. Una limitación es el factor económico, debido al alto costo de las campañas de esta naturaleza.

\section{Sistemas de valores y creencias}

Como se ha indicado anteriormente, el CSPA está básicamente sustentado en la habilidad de iniciar una acción con el propósito de promover y preservar la salud, estimulando de esta manera un estado de equilibrio mental y físico que refleje, en el análisis final, un estilo de vida óptimo $y$ satisfactorio.

La habilidad de iniciar acción, está en función a la premisa básica de la teoría de la acción. De acuerdo con este postulado, el individuo interpreta su propia conducta en términos de conceptos tales como intencionalidad, planes y metas, en base a un sistema de valores y creencias. En las páginas siguientes, voy a intentar hacer un análisis cognitivo de este proceso. 
Dos conceptos importantes en el proceso de iniciar una acción son los sistemas de valores y creencias. Realmente éstos dirigen y controlan la conducta humana. La definición conceptual de los valores está caracterizada por tres aspectos de contenido: (a) el tipo de meta que persiguen, (b) el interés al que sirven, y (c) el carácter motivacional.

El ser humano aprende a "valorar" diferentes emociones a diferentes niveles de intensidad, los cuales están supuestamente dirigidos a crear estados de placer. Cada persona mantiene una jerarquía de valores, los cuales dirigen su enfoque mental de manera constante. La atención que la persona presta a los eventos y circunstancias en su vida diaria, están fundamentalmente basados en esta sistema de valores (Rokeach, 1973). Estos valores indican lo que es más importante para cada persona, y qué tipo de acciones van a generar mayor placer. Es por esta razón que la atención está dirigida a ese tipo de acciones con el simple propósito de obtener placer. A estos valores se denominan valores de acercamiento (v.g., amor, poder, éxito, felicidad, seguridad).

Por otro lado, existen otro tipo de valores que van a atraer la atención por el hecho de que causan dolor. Por lo general, este segundo tipo de valores impulsan a "fijar la atención" en determinadas circunstancias de la vida cotidiana con el propósito justamente de evitar dolor, por lo tanto "es necesario" evitarlos de cualquier manera. A éstos se les denomina valores de retirada. Estos valores crean estados emocionales tales como hostilidad, frustración, cólera y resentimiento. El individuo trata de evitar estos estados emocionales debido a que causan dolor.

Es importante entender que estos dos tipos de valores tienen una jerarquía, la cual es evaluada y valorada por cada individuo en base a prioridades que cada uno tiene en su vida (Schartz y Bilsky, 1987). Estas prioridades han sido creadas por cada persona en el transcurso de su vida, en base a la educación e influencia de los padres, profesores, amigos, colegas $y$, en forma general, por el medio cultural (Feather, 1975).

Un cambio en el sistema de valores representa un cambio en las prioridades de la vida del individuo; ello significa un cambio en la manera cómo observa su mundo interno y externo, en la manera cómo se autoevalúa $y$, por último, significa un cambio en su estilo de vida. 
Es muy común vivir en conflicto con el propio sistema de valores; cuando ello ocurre se experimentan serias limitaciones, particularmente en relación a las metas y bienestar personal. Este conflicto conduce a un autosabotaje de los planes, metas, y acciones. Obviamente, este proceso no ocurre en el individuo a un nivel consciente. Por otro lado, es importante entender el significado y poder que tiene este sistema de valores. Uno de los aspectos más importantes es saber cuáles son y lo que representan para uno.

La razón por la cual el individuo necesita conocer sus valores, es básicamente, para comprender la naturaleza de su enfoque mental. Esta atención que uno fija en ciertos eventos determina el estado emocional del individuo en ese momento, lo cual es crucial para el inicio de la acción como lo veremos más adelante. Si la persona logra modificar ciertos valores, será capaz de cambiar el curso de su atención. Ello significa cambiar el curso de sus acciones, por lo tanto, de su fururo.

Para que este cambio conductual ocurra, lo primero que se necesita saber es cuáles son esos valores, y comenzar a analizar cuáles son los que crean conflicto (Schwartz y Inbar-Saban, 1988). Un ejemplo típico de conflicto de valores se da en individuos que tienen como prioridad lograr el éxito personal, sin embargo, la salud no la consideran importante. Es difícil poder concebir a una persona que abusa del consumo de alcohol y drogas, tratando de ser un individuo de éxito.

Los valores no son permanentes. Ellos son constantemente afectados por el medio cultural, medios de comunicación masiva y otros factores sociales (Bond, 1988). Lo importante es mantener conciencia de estos cambios constantes, de tal manera que éstos sirvan a los propósitos del individuo y no representen una limitación en su conducta en el inicio de la acción y el logro de sus metas.

El sistema de creencias es el conjunto de generalizaciones o reglas creadas por uno mismo en base a la propia experiencia, acerca de lo que es necesario para poder iniciar una acción y obtener resultados positivos. Dos personas pueden tener los mismos valores, sin embargo, actuar en forma muy diferente debido a sus propios sistemas de creencias. Es decir, un conjunto de reglas que difieren entre uno y otro. 
Por ejemplo, José y Alberto tienen como prioridad lograr el éxito personal (sistema de valores). Basado en el sistema de creencias de José, este éxito puede ser reconocido como tal si ganara un sueldo anual de cincuenta mil dólares y mantuviera una cuenta de ahorros de por lo menos cien mil dólares. Para Alberto, el éxito personal está basado en el hecho de ser padre de cinco niños, mantener una buena relación con su esposa, y así lograr formar un hogar feliz. Como observamos, ambos individuos buscan lo mismo (éxito personal), basado en diferentes reglas.

Uno de los problemas más comunes que las personas experimentan en relación al sistema de creencias, es que el conjunto de reglas y generalizaciones que estos mismos individuos crean muchas veces sabotean sus posibilidades para iniciar acción y alcanzar un conjunto de metas que les permitan gozar de un estilo de vida óptimo. Ciertas personas viven con un sistema de valores y creencias creados por personas ajenas a ellos mismos, de tal manera que no logran gozar de una vida congruente y un estilo de vida satisfactorio. En esos casos no sorprende ver a estos individuos en estados constantes de hostilidad, ansiedad, y depresión crónica, evadiendo la realidad a través del excesivo consumo de alcohol y drogas.

Los sistemas de valores y creencias tienen una influencia enorme en la calidad de vida de una persona, su habilidad para iniciar acciones y la forma como afronta las situaciones de estrés. Asimismo, la habilidad con la que se obrienen resultados positivos que lleven al éxito personal está profundamente influenciada por el sistema de creencias. Al igual que los valores, el sistema de creencias de una persona no es permanente. Estas creencias varían en función a las experiencias diarias y el medio cultural del individuo.

\section{Temor al fracaso y autosabotaje}

El temor es una variable cognitiva de gran impacto negativo en el inicio de la acción y en el desarrollo del CSPA. Temor al fracaso al igual que temor al éxito, o aún más, temor al rechazo, son estados mentales que limitan las posibilidades de iniciar acciones que culminen en la adquisición del CSPA. Estas interpretaciones cognitivas creadas por uno mismo implican serias limitaciones para el cambio conductual. 
El temor es un estado mental bajo el cual ciertas estructuras cerebrales son activadas con la intención de evitar dolor emocional. Es un estado de preocupación excesiva que excita el sistema nervioso central y autónomo, produciendo un número de reacciones conductuales negativas. Por último, el temor moviliza los más profundos recursos de un individuo, los que determinan la reacción de "volar o pelear", siendo esta reacción un mecanismo fundamental de adaptación al medio ambiente (Cannon, 1939).

El impacto negativo del temor en el desarrollo del CSPA radica en el hecho de percibir y evaluar este temor de una manera intensa y extrema. Esta situación origina una serie de limitaciones para el individuo en circunstancias donde dicho temor no justifica tal reacción. Es decir, crear un estado de pánico sin que exista la real necesidad de que esto ocurra. En la mayoría de casos, tal temor es elaborado por el propio individuo sin una justificación real, creando así una situación de vulnerabilidad en sí mismo.

El temor al fracaso representa el mayor obstáculo en el proceso de iniciar una acción. Al igual que el estrés, el temor al fracaso es, únicamente, una interpretación que tiene un significado, por lo común negativo, para cada persona. Por lo general, el grado de repercusión psicológica también varía de acuerdo a cada individuo (Birney, Burdick, y Teevan, 1969).

Desde mi perspectiva personal, es imposible fracasar. Basado en el concepto de flexibilidad, si el resultado de una acción no produce los resultados positivos esperados, el plan de acción debe ser modificado, hasta aproximarse y llegar al objetivo final. En este sentido el fracaso no existe; lo único que se observa son resultados que son interpretados por el propio individuo, quien, en última instancia, es el que le otorga la etiqueta de "fracaso".

Por ejemplo, dentro del proceso de manejo de estrés utilizando técnicas de relajación, es muy común que una persona experimente con varias de las técnicas disponibles hasta que, finalmente, se adapte a la más conveniente. El hecho que una de las técnicas no tenga efectividad para este individuo, no significa que éste fracase. El considerar una posibilidad le ofrece la oportunidad de lograr sus metas planeadas. La creencia en el éxito inspira éxito y éste, a su vez, facilita en gran medida la adquisición del CSPA. 
Para generar un cambio conductual en este sentido, se requiere que el individuo modifique sus propias "reglas de juego" a través de la transformación en el significado de los conceptos de fracaso y rechazo. Cada persona tiene una opinión diferente de estos dos conceptos. El problema radica en el principio utilizado para definir cognitivamente lo que es fracaso.

El fracaso es simplemente una interpretación negativa por parte del individuo de los resultados encontrados. En la mayoría de casos, esta "regla emocional" no es más que una simple forma de autosabotaje. Si una persona experimenta temor al fracaso en forma constante, es muy posible que se desenvuelva bajo pautas que limitan seriamente sus posibilidades de iniciar una acción y vivir un estilo de vida satisfactorio. El proceso de modificar estas reglas y transformar el significado de este constructo, es uno de los pasos fundamentales para el aprendizaje del CSPA.

Otro tipo de temor que afecta enormemente a una gran mayoría de personas, es el temor al rechazo. Esta es otra limitación que uno puede experimentar en el proceso de iniciar una acción. Al igual que el temor al fracaso, este tipo de interpretación es el resultado de una evaluación personal y subjetiva la cual varía en cada individuo. Si una persona se siente rechazada, es porque ella misma ha "creado" tal sentimiento de rechazo, basado en las propias reglas y autodefiniciones acerca de este estado mental.

Otra razón por la cual una persona se priva de gozar de un estilo de vida satisfactorio, es el constante autosabotaje, que ocurre de una manera consciente o fuera de la conciencia. Esta es una de las causas de que muchos individuos no logren culminar sus metas. El autosabotaje es una conducta que ocurre con el propósito de evitar el dolor supuestamente causado por el esfuerzo que implica alcanzar determinada meta; en este sentido es una conducta autoprotectora. Es el resultado de asociar al proceso de iniciar una acción con una situación largamente dolorosa.

Si una persona sabotea sus posibilidades de alcanzar una meta o lograr el éxito en cierto proyecto, ello se debe a que, en alguna medida, este individuo interpreta tal proceso como doloroso. Este fenómeno explica en la mayoría de casos el abandono de objetivos y metas. Cuando estas 
situaciones ocurren, el sistema nervioso, de alguna manera y en alguna medida, asocia las metas planeadas con la posibilidad de experimentar una emoción dolorosa, en lugar de convertirse en una experiencia placentera. Por lo tanto, se actúa de una manera autodestructiva y de sabotear lo conseguido hasta ese momento.

Para eliminar el autosabotaje del repertorio conductual, se requiere modificar las asociaciones, particularmente en cuanto al significado de iniciar acción. En este proceso de cambiar tales asociaciones, es necesario modificar cognitivamente el sistema de creencias de uno.

Figura 3: Factores de influencia sobre el Comportamiento Social Pro Acrivo

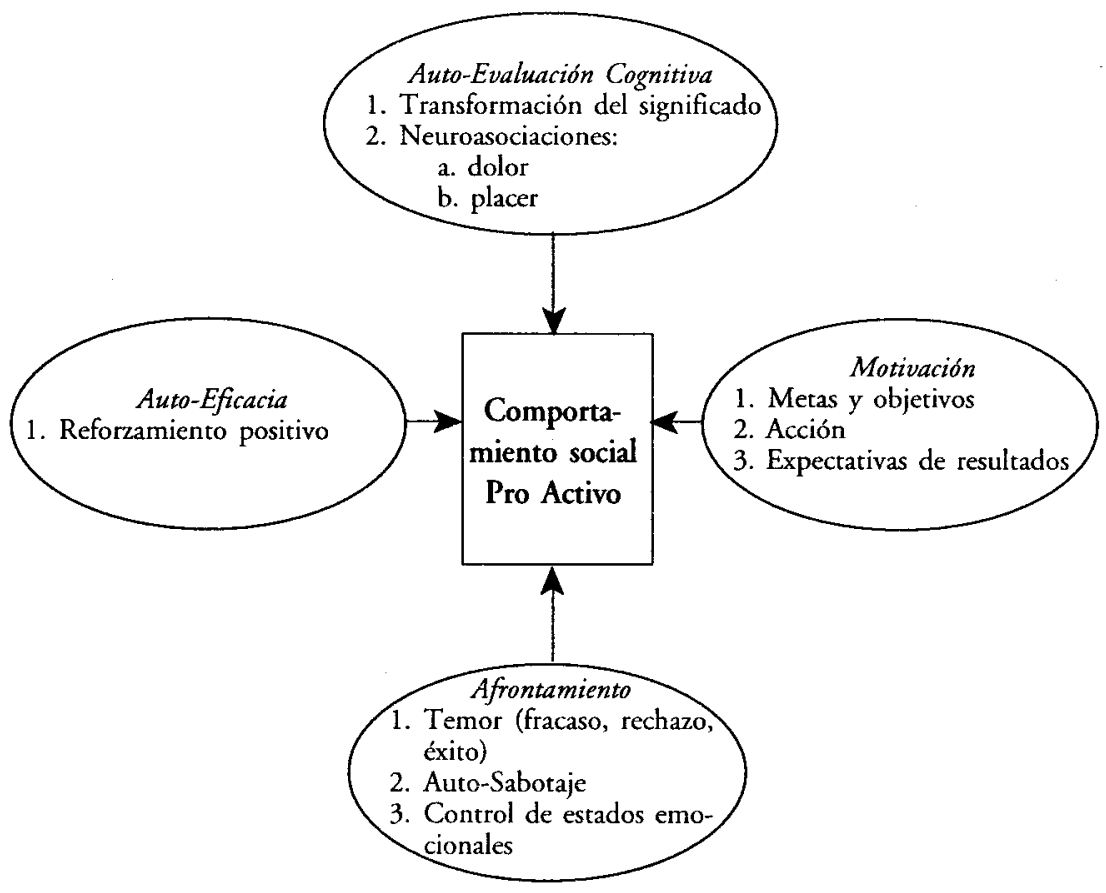




\section{De la emoción a la acción}

Dentro del proceso de desarrollo del CSPA y del inicio de la acción, es vital aprender a manejar y controlar los estados emocionales. La manera como uno se siente emocionalmente en cada momento es el resultado de cómo dirigimos nuestro pensamiento y nuestra propia fisiología.

Es importante ser consciente de la necesidad de activar el sistema fisiológico para el buen manejo y control de los estados emocionales. La manera como se mueven los músculos, gestos, la forma como una camina, la postura del cuerpo, el tono y la velocidad del lenguaje, determinan de una manera significativa cómo una persona se siente en cada momento. Otro aspecto fisiológico es el aprendizaje de adecuados hábitos alimenticios. Particularmente el azúcar, los productos que contienen cafeína, y ciertos carbohidratos complejos ocasionan un cambio dramático en el metabolismo, lo cual a su vez genera notables fluctuaciones del estado emocional.

La forma más importante de manejar los estados emocionales es dirigiendo y controlando la atención mental, es decir, el enfoque del pensamiento y la atención que uno presta a situaciones del diario vivir. La habilidad de poder controlar esta atención, está basado en dos aspectos fundamentales: En primer lugar, ser consciente en que se fija tal atención. En segundo lugar, ser consciente de cómo se fija dicha atención, es decir el contexto del evento. Si uno aprende a manejar, dirigir y controlar estos dos aspectos fundamentales del enfoque mental representados por: qué y cómo, uno tendrá mejores posibilidades de controlar sus estados emocionales, y por lo tanto su propia conducta.

La calidad del estilo de vida de una persona está basada en la calidad de los estados emocionales que ésta experimenta cada día. La manera como uno se siente momento a momento, determina, en gran medida, la forma como uno trata a sí mismo y a sus semejantes. En este sentido, el desarrollo del CSPA depende de un equilibrio emocional basado en la habilidad del individuo de crear un estado de satisfacción, el cual le permita madurar $y$ autorealizarse.

Esta posibilidad de crear un estado de satisfacción constante está críticamente en función al enfoque mental y a la forma como uno presta 64 
atención a los eventos de la vida diaria. Lo que realmente determina este enfoque mental, es la autoevaluación de cada una de los eventos situacionales que vivimos, sean estos "estresores" o simplemente experiencias comunes. Me gustaría señalar claramente que el significado que uno le otorga a los estresores o eventos circunstanciales, va a determinar cómo nos sentimos.

Las autoevaluaciones son, básicamente, preguntas que en forma automática el individuo se hace durante el proceso de pensar o autocomunicarse. Estas "preguntas" tienen como intención averiguar: qué significa tal circunstancia para mí, y qué debo hacer en este caso. Las respuestas que se den a tales preguntas van a determinar el estado emocional y conducta. Por ejemplo, si generalmente nos sentimos deprimidos, ello ocurre porque, por lo común, nos formulamos "preguntas" que nos hacen sentir deprimidos.

Podemos considerar que la calidad del estilo de vida de una persona está en función a la calidad de preguntas que ésta se haga a cada momento. Si esas preguntas están planteadas dentro de un contexto positivo, es muy posible que sea un individuo satisfecho y que goza de su propio estilo de vida. De otra manera, las posibilidades de experimentar cólera, hostilidad, ansiedad y pérdida de la salud son bastante reales (Moscoso, 1995; Moscoso y Oblitas, 1994, Spielberger y Moscoso, 1995, Spielberger y Moscoso, en prensa).

Estamos de acuerdo en aceptar que los estados emocionales negativos no ocurren por accidente o casualidad. Ansiedad, cólera, hostilidad y depresión no son estados o síntomas que una persona adquiere por contagio; tampoco son el resultado de bacterias. Para que una persona se sienta de una u otra manera tiene que "pensar" de cierta forma, y enfocar su mundo desde cierta perspectiva. El tratar de entender y explicar este proceso es, ciertamente, uno de los objetivos de la terapia cognitiva.

Un buen número de estudios de investigación en el campo de la salud, depresión, autoestima, cólera y hostilidad sugieren que las evaluaciones causadas por eventos negativos, son la causa de conductas desajustadas (Abramson, Seligman, y Teasdale, 1978; Bargh y Tota, 1988; Brockner, 1979; Carver y Ganellen, 1983; Carver, Ganellen, y Behar-Mitrani, 1985, Epstein y Meier, 1989, Moscoso, 1995c). Esto no nos sorprende. Sin 
embargo, otros estudios indican que la depresión, una pobre autoestima, y el patrón de un pensamiento negativista podrían ser básicamente desórdenes de tipo motivacional (Peterson y Seligman, 1984). Ciertamente, este último postulado tiene gran relevancia sobre los procesos cognitivos que limitan el inicio de la acción y el desarrollo del CSPA.

Asimismo, encuentro sumamente fascinante el análisis que la teoría cognitiva experiencial del sí mismo (Epstein, 1980, 1990, 1991a) hace en relación a las autoevaluaciones negativas por parte del individuo, sus implicancias en el desarrollo de la autoestima, y la habilidad de iniciar una acción. De acuerdo con Epstein (1983, 1991b), las autoevaluaciones son procesos que se generan fuera de la conciencia. El individuo, por lo común, no tiene la intención de crear su propio sufrimiento. Sin embargo, por razones que están fuera de su conciencia, éste interpreta situaciones del diario vivir, maneja sus estados emocionales y dirige su conducta de tal manera que sacrifica el desarrollo de su autoestima con el único propósito de satisfacer otras necesidades más apremiantes.

Esta teoría postula que la conducta del ser humano está dirigida en base a dos necesidades fundamentales: La necesidad de maximizar placer y minimizar dolor, así como la necesidad de proteger la autoestima. Debido a que la conducta del individuo supone el mantener una especie de armonía entre la necesidad de maximizar placer y minimizar dolor, en la cual la persona otorga prioridad al evitamiento del dolor, ocurre lo siguiente:

Si este equilibrio se encuentra en peligro, el individuo optará por "sacrificar" la protección de su autoestima. El mecanismo por el cual el individuo procesa esta información, de manera que ororgue prioridad al evitamiento del dolor en relación a la obtención de placer, explica claramente por qué algunas personas están motivadas a mantener una actitud negativista sacrificando el desarrollo de su autoestima y por lo tanto creando serias limitaciones para el inicio de la acción.

Las evaluaciones negativas son susceptibles de control. Estas pueden ser eliminadas en base a técnicas de terapia cognitiva y de programas de condicionamiento (Moscoso, 1993a, 1994b). No es mi intención en este artículo discutir detalles de sus aplicaciones clínicas, sin embargo, considero conveniente hacer un breve comentario acerca del Condicionamiento 
Neurofisiológico Asociativo (CNA), como una técnica terapéutica que permite el manejo y control de los estados emocionales.

Programas de condicionamiento neuropsicofisiológico vienen siendo utilizados desde los inicios del condicionamiento clásico (Pavlov, 1927). En las últimas décadas, estas técnicas se encuentran altamente sofisticadas y son aplicadas con magníficos resultados en las áreas de psicología de la salud y psicología deportiva.

Los resultados de este tipo de programas son típicamente observados en atletas y deportistas en general, quienes aprenden estrategias de condicionamiento que les permiten no solamente el manejo exitoso de sus estados emocionales, vital para los objetivos que se proponen, sino también tener la habilidad de accionar, tomar decisiones y lograr las metas planeadas.

Uno de los factores cruciales para el éxito, en términos de lograr un cambio conductual a través del CNA, es el grado de compromiso por parte del individuo, de tener realmente la intención y deseo sincero, así como también posibilitar y crear tal cambio.

Las premisas básicas del condicionamiento Neurofisiológico Asociativo son los siguientes:

1. Lograr el estado mental de crear que algo debe cambiar dentro de uno mismo; uno mismo debe de producir ese cambio; y, por último, uno mismo puede producir ese cambio en este mismo momento.

2. Interrumpir el patrón de pensamiento o de evaluación negativa. Cuanto más frecuente sea la interrupción de los patrones de pensamiento y de evaluación que desajustan el estado emocional y la conducta, el sentimiento de control personal será mayor.

3. Crear una nueva asociación, utilizando el principio de placer y dolor como reforzadores. Esta nueva asociación es creada con el propósito de motivar una nueva forma de pensar, de evaluar los estresores y eventos y circunstancias de la vida diaria y, en última instancia, crear nuevas conductas que faciliten un estilo de vida óptimo. 
Un aspecto importante dentro del proceso CNA es la utilización de la intensidad del nuevo estado emocional del individuo durante el entrenamiento clínico. Esta intensidad es vital en la producción de las nuevas asociaciones condicionadas, así como en la preservación de éstas a través del tiempo. Este programa de entrenamiento terapéutico es de gran aplicabilidad en la adquisición del CSPA y en la creación de nuevos estilos de vida.

\section{Sumario y conclusiones}

En este estudio se hace un análisis del Comportamiento Social Pro Activo, el que se entiende como un patrón conductual caracterizado por el conjunto de actitudes, hábitos y conductas que promueven y estimulan la salud del individuo. Este patrón conductual se desarrolla en función al planeamiento de metas, al inicio de la acción y la evaluación permanente de los resultados parciales obtenidos.

Se revisa el concepto de autoeficacia y se indica su importancia en el desarrollo del Comportamiento Social Pro Activo. Se hace referencia a variables de tipo cognitivo, tales como los sistemas de valores y creencias, los cuales facilitan el proceso de la acción. Se entienden estos sistemas de valores y creencias como las fuerzas que dirigen y controlan la conducta humana.

Por otro lado, se hace un análisis de los factores que tienen un efecto limitante sobre este mismo proceso de la acción. En este sentido, se establecen distinciones entre el temor al fracaso, al éxito, al rechazo, y su relación con el fenómeno del auto-sabotaje. Se señala la importancia de la transformación del significado y sus beneficios en la extinción del temor.

Se presenta un modelo conceptual acerca del control de los estados emocionales y sus implicancias en el proceso de la acción. Se considera a los estados emocionales como el resultado de autoevaluaciones que el individuo elabora a cada momento. Asimismo, del control sobre sus funciones fisiológicas.

Finalmente, se hizo una breve descripción de las premisas fundamentales del Condicionamiento Neurofisiológico Asociativo como un programa terapéutico que facilita el manejo de los estados emocionales del individuo. 


\section{Referencias}

Abramson, L.Y., Seligman, M.E.P., \& Teasdale, J.D. (1978). Learned helplesseness in humans: Critique and reformulation. Journal of Personality and Social Psychology, 87, 49-74.

Ahrens, A.H. (1978). Theories of depression: The role of goals and the self-evaluation process. Cognitive Therapy and Research, 6, 665-680.

Bandura, A. (1977a) Self-efficacy: Toward a unifying theory of behavioral change. Psychological Review. 85, 1919-2150.

Bandura, A. (1988a). Perceived self-efficacy: Exercise of control through self-belief. In J.P. Dauwalder, M. Perrez, \& V. Hobi (Eds.), Anual series of European research in behavior therapy, vol, 2, pp. 27-59, Lisse, The Netherlands: Wets \& Zeitlinger.

Bandura, A. (1988b). Self-efficacy conception of anxiety. Anxiety Research, 1, 77-98.

Bandura, A. (1989a). Human agency in social cognitive theory. American Psychologist, 44, 1175-1184.

Bandura, A. (1989b). Self-regulation of motivation and action through internal standards and goal systems. In L.A. Pervin (Ed.), Goal concepts in personality and social psychology, pp. 19-85). Hillsdale, NJ: Erlbaum. Bandura, A., \& Adams, N.E. (1977). Analysis of self-efficacy theory of behavioral change. Cognitive Therapy and Research, 1, 287-308.

Bandura, A., Adams, N.E. \& Beyer, J. (1977). Cognitive processes mediating behavioral change. Journal of Personality and Social Psychology. 35, 125139.

Bandura, A., \& Cervone, D. (1983). Self-evaluative and self-efficacy mechanisms governing the motivational effects of goal systems. Journal of Personality and Social Psychology, 45, 1017-1028.

Bandura, A., \& Cervone, D. (1986). Differential engagement of selfreactive influences in cognitive motivation. Organizational Behavior and Human Decision Processes, 38, 92-113.

Bargh, J.A. \& Tota, M.E. (1988). Context-dependent automatic processing in depressión. Accesssibility of negative constructs with regard to self but not others. Journal of Personality and Social Psychology, 54, 925939.

Birney, R.C., Burdick, H, \& Teevan, R.C. (1969). Fear of failure. New York: Nostrand. 
Bond, M. (1988). Finding universal dimensions of individual variation in multicultural studies of values: The Rokeach and Chinese Value Surveys. Journal of Personality and Social Psychology, 55, 1009-1015.

Brockner, J. (1979). The effects of self-esteem, success, failure, and selfconsciousness on task performance. Journal of Personality and Social Psychology, 87, 1732-1741.

Cannon, W.B. (1939) The wisdom of the body, 2nd edition. New York: W. W. Simon.

Carver, C.S. \& Ganellan, R.J. (1983). Depression and components of selfpunitiveness; High standards, self-criticism, and overgeneralization, Journal of Abnormal Psychology, 31, 674-685.

Carver, C.S., \& Scheier, M.F. (1990). Principles of self-regulation: Action and emotion. In E.T. Higgins \& R.M. Sorrentino (Eds.) Motivation and cognition: Foundations of social behavior. vol, 2, pp. 527-561. New York: Guilford Press.

Epstein, S. (1980). The self-concept: A review and the proposal of an integrated theory of personality. In E. Staub (Ed.). Personality: Basic issues and current research, pp. 82-132. Englewood Cliffs, NJ: PrenticeHall.

Epsteins, S. (1983). The unconscious, the preconscious and the selfconcept. In J. Suls \& A. Greenwald (Eds.), Psychological perspectives on the self, vol. 2, pp. 219-247. Hillsdale, NJ: Erlbaum.

Epsteins, S. (1990). Cognitive-experimental selft-theory: An integrative theory of personality. In R. Curtis (Ed.), The relational self: Convergences in psychoanalysis and social psychology, pp. 111-137. New York: Guilford Press.

Epsteins, S. (1991a). Cognitive-experimental self-theory: An integrative theory of personality. In R. Curtis (Ed.), The relational self. Convergences in psychoanalysis and social psychology, pp. 111-137. New York: Guilford Press.

Epsteins, S. (1991b). Cognitive-experimental self-theory: Implications for developmental psycology. In M.G. Gunnar \& L.A. Sroufe (Eds.), Selfprocesses in development, vol. 23. Minnesota Symposium on Child Psychology series. Hillsdale, NJ: Erlbaum.

Epstein, S., \& Meier, P. (1989). Constructive thinking: A broad coping variable with specific components, Journal of Personality and Social Psychology, 57, 332-350. 
Feather, N.T. (1975). Values in education and society. New York: Free Press.

Lambert, M.J, Shapiro, D.A. \& Bergin, A.E. (1986). The effectiveness of psychotherapy. In S.L. Garfield \& A.E. Bergin (Eds.). Handbook of psychotherapy and behavior change (3rd ed.), New York: Wiley.

Marlett, G.A. Baer, J.S., Donovan, D.M., \& Divlahan, D.R. (1988). Addictive behavior: Etiology and treatment. Annual Review of Psychology. 39, 223-252.

Moscoso, M.S. (1993, July). Cognitive-Behavioral Stress Management for Asymptomatic and Symptomatic HIV Seropositive Individuals in USA. A Review of Data. Paper presented at the 24th Interamerican Congress of Psychology, Santiago, Chile.

Moscoso, M.S. (1994, June). Terapia Cognitivo-Conductual con Pacientes HIV-SIDA Positivos-Aspectos Clínicos y de Investigación. Paper presented at the 8th Latin American Congress of Analysis and Behavior Modification, La Paz, Bolivia.

Moscoso, M.S. (1994). La Psicología de la salud: Un enfoque multidisciplinario acerca del estrés y cambio conductal. Revista de Psicologia, 12, 47-71.

Moscoso, M.S. (1995, July). Expression and control of anger on coronary heart disease: The issue of measurement. Paper presented at the 25th Interamerican Congress of Psychology, San Juan, Puerto Rico.

Moscoso, M.S. (1995). Manejo cognitivo-conductual del estrés en individuos VIH-1 seropositivos. Avances en Psicologia Clinica Latinoamericana, 13, 39-53.

Moscoso, M.S., \& Oblitas, L.A. (1994). El proceso de lograr un estilo de vida saludable. Revista Interdisciplinaria Extensiones. 1, 3-20.

Pavlov, I.P. (1927) Conditioned reflexes. Translated by G.V. Anrep. London: Oxford University Press.

Peterson, C., \& Seligman, M.E.P. (1984). Causal explanations as a risk factor for depression: Theory and evidence. Psychological Review, 91, 347-374.

Rokeach, M. (1973). The nature of human values. New York: Free Press. Scheier, M.F., Matthews, K.A., Owens, J.F., Magovern, Sr., G.J., Lefebvre, R., Abbott, R.C., \& Carver, C.S. (1989). Dispositional optimism and recovery from coronary artery bypass surgery: The beneficial effects 
of optimism on physical and psychological well-being. Journal of Personality and Social Psychology, 57, 1024-1040.

Scheier, M.F. \& Carver, C.S. (1992). Effects of optimism on psychological and physical well-being: Theoretical overview and empirical update. Cognitive Therapy and Research, 16, 201-228.

Schwartz, S.H., \& Bilsky, W. (1987). Toward a psychological structure of human values. Journal of Personality and Social Psychology. 53, 550562.

Schwartz, S.H. \& Inbar-Saban, N. (1988). Value self-confrontation as a method to aid in weight loss. Journal of Personality and Social Psychology, 54, 396-404.

Seligman, M.E.P. (1995). The effectiveness of psychotherapy. American Psychologist, 50, 965-974.

Spielberger, C.D. \& Moscoso, M.S. (1995). La expresión de cólera y hostilidad y sus consecuencias en el sistema cardiovascular. Revista de Psicología Contemporánea, 2, 32-43.

Spielberger, C.D. \& Moscoso, M.S. (En prensa). Reacciones Emocionales del Estrés: Ansiedad y Cólera. Revista Latinoameriana de Psicologia. Wood, R.E., Bandura, A., \& Baily, T. (1990). Mechanisms governing organizational productivity in complex decision-making environments, Organizational Behavior and Human Decision Processes, 46, 181-201. 